\title{
EXTRAÇÃO DE HIDROCARBONETOS AROMÁTICOS VIA EXTRAÇÃO LÍQUIDO-LÍQUIDO: DADOS EXPERIMENTAIS E MODELAGEM TERMODINÂMICA
}

\author{
F. M. R. MESQUITA ${ }^{1}$, R. S. PINHEIRO ${ }^{1}$, A. M. M. BESSA ${ }^{1}$, H. de B. SANT'ANA ${ }^{1}$ e R. S. \\ de SANTIAGO-AGUIAR ${ }^{1}$
}

${ }^{1}$ Universidade Federal do Ceará, Departamento de Engenharia Química E-mail para contato: fmrm03@gmail.com

\begin{abstract}
RESUMO - Hidrocarbonetos alifáticos são comumente encontrados em misturas multicomponentes associados a compostos aromáticos. Dada à proximidade de ponto de ebulição, o processo de separação baseado na operação unitária de destilação torna-se inviável. Por este motivo, o uso da técnica extrativa por intermédio da extração liquido-líquido tem sido comumente utilizada. O presente trabalho tem como objetivo estudar o equilíbrio liquido-liquido (ELL) de sistemas ternários constituídos de compostos alifáticos (hexano/decano) + aromático (tolueno) + sulfolano na temperatura de $30^{\circ} \mathrm{C}$. Além da obtenção de dados experimentais, também foi realizada modelagem termodinâmica utilizando os seguintes modelos de coeficiente de atividade: NRTL, UNIQUAC e UNIFAC para um conjunto de dados experimentais que totalizam 17 sistemas ternários (alifático + aromático + sulfolano), dois destes sistemas foram obtidos neste trabalho; e, outros 15 foram obtidos da literatura, apresentando desvio médio quadrático global de $1,08 \%, 1,16 \%$ e $3,55 \%$, respectivamente. Estes resultados indicam que os modelos correlacionaram de maneira satisfatória os dados experimentais.
\end{abstract}

\section{INTRODUÇÃO}

Benzeno, tolueno e xileno, conhecidos como BTX's, são compostos orgânicos aromáticos extremamente importantes na indústria petroquímica. Esses compostos apresentam alta toxicidade, sendo o benzeno carcinogênico e mutagênico fraco. Por este motivo, o tolueno tem sido preferível para aplicações industriais (Sevgili, 2010). Hidrocarbonetos aromáticos são geralmente encontrados em misturas multicomponentes com hidrocarbonetos alifáticos. Dada a proximidade de pontos de ebulição, o uso da operação unitária de destilação torna-se inviável. Por este motivo, a separação desses componentes é, usualmente realizada por intermédio da extração líquido-líquido.

O projeto, a operação e a otimização dos processos de extração requerem o uso de modelos termodinâmicos eficientes para descreverem o equilíbrio líquido-líquido. Vários modelos foram desenvolvidos para atender a essa exigência, dentre os quais os modelos de composição local. No entanto, o uso desses modelos requer o conhecimento de parâmetros de interação binária que ainda são escassos para sistemas característicos de extração de aromáticos. Vale salientar que, dados experimentais confiáveis de ELL são necessários para a 
determinação dos parâmetros supramencionados.

Desta forma, o objetivo do presente trabalho foi a determinação de dados de ELL para dois sistemas ternários, a saber: hexano + tolueno + sulfolano e decano + tolueno + sulfolano, à pressão atmosférica e temperatura de $30^{\circ} \mathrm{C}$. Os modelos termodinâmicos NRTL, UNIQUAC e UNIFAC foram aplicados a um conjunto de dados composto de 17 sistemas ternários constituídos de compostos alifáticos, aromáticos e sulfolano, dois desses sistemas determinados neste trabalho. Os modelos NRTL e UNIQUAC foram correlacionados com os dados experimentais e foram determinados novos valores de parâmetros de interação binária entre os componentes dos sistemas. Além disso, a capacidade preditiva do modelo UNIFAC foi avaliada.

\section{METODOLOGIA}

\subsection{Materiais}

Decano, hexano, tolueno e sulfolano fornecidos pela Sigma-Aldrich (grau de pureza superior a 99,9\%) foram utilizados sem nenhuma etapa de purificação prévia. Análises de densidade foram realizadas por intermédio de um densímetro (Anton Paar, modelo DSA 5000).

\subsection{Determinação Experimental de Dados de Equilíbrio Líquido-Líquido}

Dados de ELL foram determinados para os sistemas ternários hexano (1) + tolueno (2) + sulfolano (3) e decano (1) + tolueno (2) + sulfolano (3) a $30^{\circ} \mathrm{C}$. A fim de determinar a região bifásica, foram construídas curvas binodais através do método titulométrico. $\mathrm{O}$ procedimento foi realizado em células de equilíbrio, onde foram previamente preparadas misturas binárias e um terceiro componente era adicionado até que fossem observados alterações macroscópicas do sistema. Em seguida, foram coletadas amostras da mistura ternária, de composição conhecida, para análise de densidade. A partir da correlação entre a fração mássica e a densidade, foram construídas curvas de calibração para posterior quantificação das fases em equilíbrio.

Uma vez delimitada a região heterogênea dos sistemas hexano + tolueno + sulfolano e decano + tolueno + sulfolano a $30^{\circ} \mathrm{C}$, foram estimados pontos de composição global dentro desta região. Estes pontos foram reproduzidos em células de equilíbrio. Após a preparação das misturas de composição global, o sistema foi agitado vigorosamente por 3 horas e logo em seguida submetido a um repouso de 16 horas, tempo suficiente para que ocorresse a separação das duas fases e a formação de um interface bem definida. A composição das fases em equilíbrio foi quantificada pelo método de densimetria através de uma equação polinomial de segundo grau, maiores detalhes da técnica de quantificação estão disponíveis em Mesquita et al. (2012).

\subsection{Modelagem Termodinâmica}

Os modelos termodinâmicos de composição local, NRTL (Renon e Prausnitz, 1968) e UNIQUAC (Abrams e Prausnitz, 1975), foram usados para correlacionar dados experimentais de sistemas ternários. Os dados de ELL para os sistemas estudados foram preditos usando o 
modelo de contribuição de grupo UNIFAC (Fredenslund et al., 1977). Os sistemas avaliados estão apresentados na Tabela 1.

Tabela 1 - Sistemas experimentais obtidos na literatura para a modelagem termodinâmica

\begin{tabular}{lcl}
\hline \multicolumn{1}{c}{ Sistema } & $\begin{array}{c}\text { Número de } \\
\text { tie-lines }\end{array}$ & \multicolumn{1}{c}{ Referência } \\
\hline Sulfolano + etilbenzeno + ciclohexano & 7 & Ashour e Abu-Eishah, 2006b. \\
Sulfolano + benzeno + ciclohexano & 7 & Ashour e Abu-Eishah, 2006a. \\
Sulfolano + tolueno + ciclohexano & 7 & Ashour e Abu-Eishah, 2006a. \\
Sulfolano + cumeno + ciclohexano & 6 & Ashour e Abu-Eishah, 2006a. \\
Sulfolano + benzeno + 1-hepteno & 10 & Chen et al., 2000b. \\
Sulfolano + tolueno + 1-hepteno & 10 & Chen et al., 2000b. \\
Sulfolano + benzeno + hexano & 10 & Chen et al., 2000a. \\
Sulfolano + tolueno + hexano & 10 & Chen et al., 2000a. \\
Sulfolano + xileno + hexano & 10 & Chen et al., 2000a. \\
Sulfolano + benzeno + octano & 10 & Chen et al., 2000a. \\
Sulfolano + tolueno + octano & 10 & Chen et al., 2000a. \\
Sulfolano + xileno + octano & 9 & Lin, 2011. \\
Sulfolano + secbutilbenzeno + decano & 8 & Lin, 2011. \\
Sulfolano + secbutilbenzeno + dodecano & 8 & Lin, 2011. \\
Sulfolano + xileno + tetradecano & 8 & Lin, 2011. \\
\hline
\end{tabular}

Os parâmetros de interação binária ajustáveis dos modelos NRTL e UNIQUAC foram estimados a partir dos dados experimentais, minimizando a função objetivo de composição, FO, através do método numérico de minimização Simplex modificado (Nelder e Mead, 1965).

$$
F . O .=\sum_{k}^{D} \sum_{j}^{M} \sum_{i}^{N-1}\left\{\left(x_{i j k}^{D, \exp }-x_{i j k}^{D, \text { calc }}\right)^{2}+\left(x_{i j k}^{S, \exp }-x_{i j k}^{S, \text { calc }}\right)^{2}\right\}
$$

A avaliação dos modelos termodinâmicos, NRTL, UNIQUAC e UNIFAC, foi realizada comparando os dados experimentais e simulados calculando o desvio quadrático médio (RMSD).

$$
r m s d=100 \sqrt{\frac{\sum_{i}^{M} \sum_{j}^{N}\left(x_{i j}^{D, \exp }-x_{i j}^{D, \text { calc }}\right)^{2}+\left(x_{i j}^{S, \exp }-x_{i j}^{S, \text { calc }}\right)^{2}}{2 M N}}
$$

\section{RESULTADOS E DISCUSSÕES}

\subsection{Dados De Equilíbrio Líquido-Líquido}

Dados de composição e densidade das misturas ternárias na curva binodal para ambos os sistemas estudados são apresentadas na Tabela 2. 
Tabela 2 - Dados da curva binodal para decano + tolueno + sulfolano e hexano + tolueno + sulfolano a $30^{\circ} \mathrm{C}$

\begin{tabular}{|c|c|c|c|c|c|}
\hline \multicolumn{3}{|c|}{$\operatorname{Decano}(1)+$ tolueno $(2)+$ sulfolano $(3)$} & \multicolumn{3}{|c|}{ Hexano $(1)+$ tolueno $(2)+$ sulfolano $(3)$} \\
\hline$w_{1}$ & $w_{2}$ & $\rho\left(\mathrm{g} \cdot \mathrm{cm}^{-3}\right)$ & $w_{1}$ & $w_{2}$ & $\rho\left(\mathrm{g} \cdot \mathrm{cm}^{-3}\right)$ \\
\hline 0,9696 & 0,0000 & 0,723375 & 0,7106 & 0,2798 & 0,713830 \\
\hline 0,8516 & 0,1283 & 0,738188 & 0,6411 & 0,3305 & 0,725765 \\
\hline 0,7228 & 0,2497 & 0,754379 & 0,5478 & 0,4394 & 0,743136 \\
\hline 0,5882 & 0,3629 & 0,778140 & 0,4619 & 0,5115 & 0,762782 \\
\hline 0,4722 & 0,4796 & 0,795209 & 0,4940 & 0,4849 & 0,755450 \\
\hline 0,3483 & 0,5662 & 0,824719 & 0,8609 & 0,1287 & 0,686473 \\
\hline 0,2095 & 0,6173 & 0,865640 & 0,3405 & 0,5727 & 0,802426 \\
\hline 0,0150 & 0,0000 & 1,255210 & 0,0099 & 0,0766 & 1,210342 \\
\hline 0,0228 & 0,1333 & 1,181130 & 0,0103 & 0,0914 & 1,202431 \\
\hline 0,0236 & 0,2482 & 1,122323 & 0,0122 & 0,1471 & 1,177899 \\
\hline 0,0310 & 0,3639 & 1,066908 & 0,0121 & 0,1831 & 1,157815 \\
\hline 0,0677 & 0,4671 & 1,011712 & 0,0189 & 0,1845 & 1,152061 \\
\hline 0,0516 & 0,5071 & 1,010779 & 0,0327 & 0,2452 & 1,114266 \\
\hline
\end{tabular}

Dados experimentais de ELL para os sistemas decano + tolueno + sulfolano e hexano + tolueno + sulfolano, juntamente com os valores do coeficiente de distribuição e seletividade, a temperatura de $30^{\circ} \mathrm{C}$ são mostrados através da Tabela 3 e da Figura 1.

Tabela 3 - Dados de ELL para decano + tolueno + sulfolano e hexano + tolueno + sulfolano a

\begin{tabular}{|c|c|c|c|c|c|c|c|}
\hline \multicolumn{2}{|c|}{ Composição Global } & \multicolumn{2}{|c|}{$\begin{array}{c}\text { Fase rica em } \\
\text { alifático }\end{array}$} & \multicolumn{2}{|c|}{$\begin{array}{c}\text { Fase rica em } \\
\text { solvente }\end{array}$} & \multirow[t]{2}{*}{$D_{2}$} & \multirow[t]{2}{*}{$S$} \\
\hline$w_{1}$ & $w_{2}$ & $w_{1}$ & $w_{2}$ & $w_{1}$ & $w_{2}$ & & \\
\hline \multicolumn{8}{|c|}{ decano $(1)+$ tolueno $(2)+$ sulfolano $(3)$} \\
\hline 0,4749 & 0,0535 & 0,8959 & 0,0976 & 0,0161 & 0,0222 & 0,23 & 12,61 \\
\hline 0,4424 & 0,1158 & 0,8059 & 0,1929 & 0,0169 & 0,0563 & 0,29 & 13,89 \\
\hline 0,4262 & 0,1510 & 0,7609 & 0,2328 & 0,0174 & 0,0742 & 0,32 & 13,90 \\
\hline 0,4066 & 0,1912 & 0,7119 & 0,2738 & 0,0182 & 0,0966 & 0,35 & 13,84 \\
\hline 0,3779 & 0,2507 & 0,6455 & 0,3265 & 0,0195 & 0,1315 & 0,40 & 13,33 \\
\hline \multicolumn{8}{|c|}{ hexano $(1)+$ tolueno $(2)+$ sulfolano $(3)$} \\
\hline 0,4674 & 0,1755 & 0,7367 & 0,2326 & 0,0067 & 0,0965 & 0,41 & 45,62 \\
\hline 0,2815 & 0,2885 & 0,6533 & 0,3032 & 0,0112 & 0,1234 & 0,41 & 23,74 \\
\hline 0,3340 & 0,2135 & 0,5450 & 0,4099 & 0,0192 & 0,1709 & 0,42 & 11,86 \\
\hline 0,2562 & 0,3322 & 0,4884 & 0,4732 & 0,0239 & 0,1998 & 0,42 & 8,62 \\
\hline 0,2453 & 0,3521 & 0,4607 & 0,5063 & 0,0255 & 0,2097 & 0,41 & 7,47 \\
\hline
\end{tabular}

A partir da Figura 1 pode ser observado que os dois sistemas estudados apresentaram uma ampla região bifásica. Essa característica é bastante positiva para a extração líquidolíquido, uma vez que evidencia a possibilidade de formação de duas fases num amplo domínio de concentrações. Adicionalmente, observa-se, a partir da inclinação das linhas de amarração, que o tolueno tem maior afinidade pela fase rica em compostos alifáticos, dada a semelhança de polaridade entre os componentes. Além disso, também pode ser visto que a fase rica em sulfolano também concentrou apreciável concentração de tolueno. 


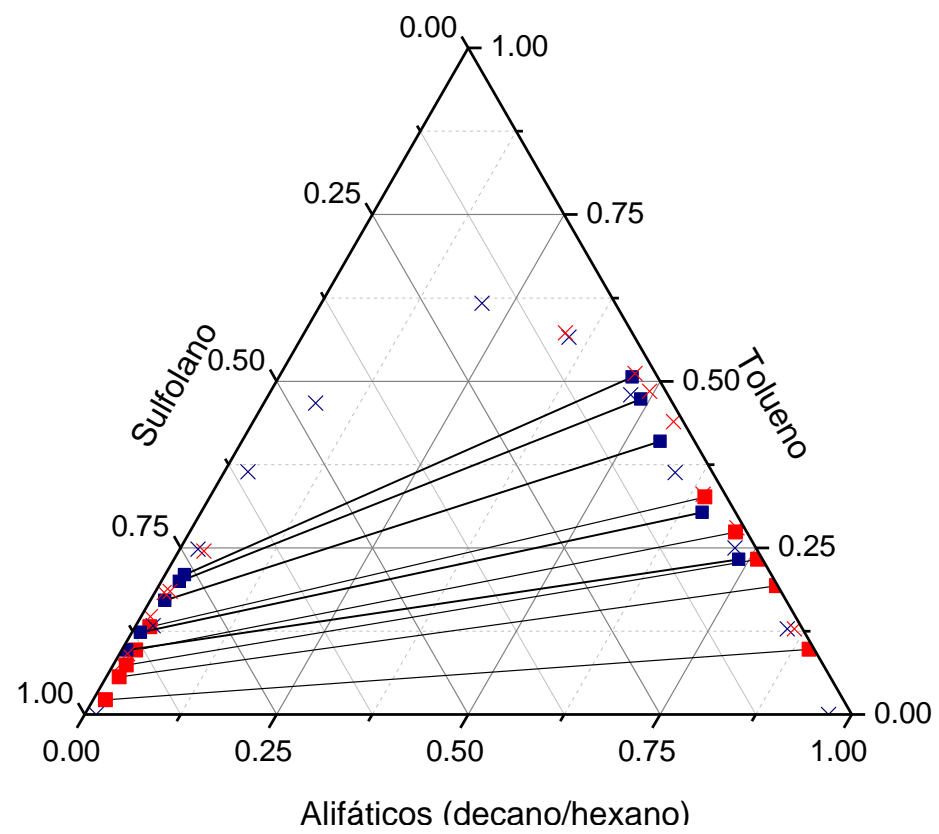

Figura 1 - Dados da curva binodal, $(\mathrm{x})$ : decano + tolueno + sulfolano e (x): hexano + tolueno + sulfolano. Dados experimentais de ELL, ( $\square)$ : decano + tolueno + sulfolano e ( $\mathbf{\square})$ : hexano + tolueno + sulfolano.

A separação do soluto entre as fases líquidas é normalmente estudada através do coeficiente de distribuição denominado $\mathrm{D}$, definido como a razão da concentração de um dos componentes do sistema nas duas fases (Sandler, 2006). Esta grandeza pode ser calculada para todas as espécies presentes em um sistema em equilíbrio utilizando a Equação 3.

$$
D_{2}=\frac{{w_{I I}}^{S E}}{{w_{I I}}^{A}}
$$

Conforme mostra a Tabela 3 os valores de coeficientes de distribuição calculados são inferiores a 1, isto indica que a separação do tolueno a baixas concentrações pode ser realizada com êxito utilizando-se o sulfolano, como agente extrator.

Outro parâmetro industrial importante é denominada de seletividade (S), que reflete a capacidade e a eficiência do processo de extração. A seletividade é calculada pela Equação 4.

$$
S=\frac{\left(w_{I I} / w_{I}\right)^{S E}}{\left(w_{I I} / w_{I}\right)^{A}}
$$

na qual, $w_{I}$ e $w_{I I}$, representam fração mássica do decano e do tolueno, respectivamente. Os sobrescritos, SE e A, denotam fase rica em solvente e fase rica em alifático. 
Observa-se na Tabela 3 que para todas as linhas de amarração o parâmetro de seletividade é maior que 1, indicando que a extração do tolueno é possível. Para o sistema contendo hexano + tolueno + sulfolano a seletividade assume valores maiores que para o sistema contendo decano + tolueno + sulfolano. Esse resultado corrobora os resultados obtidos por Letcher et al. (1996) que indica que a seletividade diminui com o aumento da cadeia carbônica do hidrocarboneto alifático.

\subsection{Modelagem Termodinâmica}

A Tabela 4 apresenta os valores dos parâmetros de interação binária, Aij e Aji, estimados pelos modelos NRTL e UNIQUAC para cada par binário.

Tabela 4 - Parâmetros de interação binária para os modelos NRTL e UNIQUAC

\begin{tabular}{lcccc}
\hline \multicolumn{1}{c}{ Sistemas } & \multicolumn{2}{c}{ NRTL } & \multicolumn{2}{c}{ UNIQUAC } \\
\hline & Aij & Aji & Aij & Aji \\
\hline Sulfolano (1) + Etilbenzeno (2) & 296,91 & 272,57 & 12,417 & 152,62 \\
Sulfolano (1) + Ciclohexano (3) & 645,21 & 2987,1 & 35,401 & 641,66 \\
Sulfolano (1) + Benzeno (4) & 3,6361 & 357,81 & 35,570 & $-10,923$ \\
Sulfolano (1) + Tolueno (5) & 72,732 & 376,96 & 55,241 & 73,259 \\
Sulfolano (1) + Cumeno (6) & 438,07 & 166,23 & $-10,983$ & 165,76 \\
Sulfolano (1) + xileno (7) & 167,99 & 415,18 & 46,623 & 123,26 \\
Sulfolano (1) + decano (8) & 1175,8 & 3758,1 & $-9,0338$ & 719,92 \\
Sulfolano (1) + dodecano (9) & 1091,3 & 1568,6 & $-14,192$ & 658,68 \\
Sulfolano (1) + 1-hepteno (10) & 847,68 & 1765,2 & 30,318 & 553,26 \\
Sulfolano (1) + hexano (11) & 773,90 & 2184,2 & 50,737 & 797,88 \\
Sulfolano (1) + octano (12) & 949,28 & 2325,3 & 18,870 & 914,61 \\
Sulfolano (1) + secbutilbenzeno (13) & 474,87 & 321,80 & 18,816 & 185,27 \\
Sulfolano (1) + tetradecano (14) & 3999,9 & 1476,6 & 32,425 & 548,91 \\
Etilbezeno (2) + Ciclohexano (3) & 184,26 & $-92,727$ & 77,541 & $-73,807$ \\
Ciclohexano (3) + Benzeno (4) & 161,29 & $-111,31$ & $-62,899$ & $-24,887$ \\
Ciclohexano (3) + Tolueno (5) & 1970,8 & $-927,93$ & 824,57 & $-332,50$ \\
Ciclohexano (3) + Cumeno (6) & $-116,69$ & 137,81 & $-3,6049$ & $-44,602$ \\
Benzeno (4) + 1-hepteno (10) & $-536,10$ & 908,66 & 287,89 & $-309,25$ \\
Benzeno (4) + hexano (11) & 275,95 & $-58,607$ & 7,4501 & $-60,871$ \\
Benzeno (4) + octano (12) & 602,12 & $-300,34$ & $-52,497$ & 7,5603 \\
Tolueno (5) + decano (8) & $-260,37$ & 452,11 & 418,07 & $-208,72$ \\
Tolueno (5) + 1-hepteno (10) & $-548,25$ & 790,11 & 308,52 & $-228,81$ \\
Tolueno (5) + hexano (11) & 214,86 & $-114,38$ & 159,50 & $-130,49$ \\
Tolueno (5) + octano (12) & 222,95 & $-159,57$ & 159,50 & $-130,49$ \\
Xileno (7) + hexano (11) & $-10,972$ & 64,664 & 189,48 & $-132,68$ \\
Xileno (7) + octano (12) & 564,00 & $-392,59$ & 97,928 & $-73,615$ \\
Xileno (7) + tetradecano (14) & 123,43 & $-826,07$ & 384,52 & $-306,25$ \\
Decano (8) + secbutilbenzeno (13) & $-782,71$ & 1422,1 & 61,037 & $-92,527$ \\
Dodecano (9) + secbutilbenzeno (13) & $-740,48$ & 1226,6 & $-8,4155$ & $-12,282$ \\
\hline & & & & \\
\hline
\end{tabular}

A Tabela 5 mostra que os dados de ELL foram satisfatoriamente correlacionados com os modelos NRTL e UNIQUAC para o cálculo do coeficiente de atividade apresentando desvio médio quadrático global de $1,08 \%$ e 1,16\%, respectivamente. Para o modelo UNIFAC foi obtido um desvio de 3,55\% mostrando que este modelo é capaz de representar o ELL de sistemas contendo hidrocarbonetos alifáticos, hidrocarbonetos aromáticos e sulfolano. 
Tabela 5 - Desvios quadráticos globais médios (\%) para os modelos NRTL, UNIQUAC e UNIFAC

\begin{tabular}{lccc}
\hline \multicolumn{1}{c}{ Sistemas } & NRTL & UNIQUAC & UNIFAC \\
\hline Sulfolano + Etilbenzeno + Ciclohexano & 0,31 & 0,34 & 1,63 \\
Sulfolano + benzeno + Ciclohexano & 0,58 & 0,68 & 1,08 \\
Sulfolano + Tolueno + Ciclohexano & 2,43 & 3,18 & 4,45 \\
Sulfolano + Cumeno + Ciclohexano & 0,23 & 0,39 & 4,05 \\
Sulfolano + benzeno + 1-hepteno & 0,77 & 1,14 & 4,43 \\
Sulfolano + tolueno + 1-hepteno & 1,87 & 1,76 & 4,82 \\
Sulfolano + benzeno + hexano & 0,69 & 0,64 & 1,01 \\
Sulfolano + tolueno + hexano & 0,47 & 0,39 & 0,94 \\
Sulfolano + xileno + hexano & 0,85 & 0,90 & 6,90 \\
Sulfolano + benzeno + octano & 0,60 & 0,55 & 0,91 \\
Sulfolano + tolueno + octano & 2,04 & 2,09 & 2,14 \\
Sulfolano + xileno + octano & 0,50 & 0,51 & 5,44 \\
Sulfolano + secbutilbenzeno + decano & 0,34 & 0,45 & 3,62 \\
Sulfolano + secbutilbenzeno + dodecano & 0,35 & 0,32 & 2,94 \\
Sulfolano + xileno + tetradecano & 1,00 & 0,22 & 1,83 \\
Sulfolano + tolueno + hexano & 0,94 & 0,96 & 1,04 \\
Sulfolano + tolueno + decano & 0,71 & 0,49 & 3,28 \\
\hline Desvio Médio Quadrático Global (\%) & 1,08 & 1,16 & 3,55 \\
\hline
\end{tabular}

Os resultados supracitados vão ao encontro do comportamento observado na literatura, no qual o NRTL apresenta melhores resultados na predição de dados de ELL para os sistemas estudados.

\subsection{Correlação de Othmer-Tobias}

A fim de avaliar a qualidade e confiabilidade dos dados experimentais foi aplicado a correlação de Othmer-Tobias (1942), Equação 5.

$$
\ln \left(\frac{1-w_{I I}^{S E}}{w_{I I}^{S E}}\right)=A+B *\left(\frac{1-w_{I}^{A}}{w_{I}^{A}}\right)
$$

Os coeficientes de correlação $a$ e $b$, juntamente com o coeficiente de regressão $\left(\mathrm{R}^{2}\right)$, foram determinados pelo método dos mínimos quadrados e são apresentados na Tabela 6 . $\mathrm{O}$ $\mathrm{R}^{2}$ dessa relações lineares assumiu valores de 0,998 e 0,997. A proximidade do coeficiente de regressão para 1 indica um bom grau de consistência dos referentes dados experimentais.

Tabela 6 - Coeficientes de correlação e de regressão para a equação de Othmer-Tobias

\begin{tabular}{ccccc}
\hline & & \multicolumn{3}{c}{ Coeficientes de Othmer-Tobias } \\
\cline { 3 - 5 } \cline { 3 - 4 } Sistemas & $a$ & $b$ & $R^{2}$ \\
\cline { 3 - 5 } Decano + tolueno + sulfolano & & 1,44045 & $-0,78585$ & 0,998 \\
Hexano + tolueno + sulfolano & & 1,12526 & $-1,22282$ & 0,997 \\
\hline
\end{tabular}

\section{CONCLUSÃO}


Os dados experimentais de ELL mostram que o aumento da cadeia carbônica do alifático não influenciou significativamente na região bifásica dos sistemas estudados. Os coeficientes de correlação de Othmer-Tobias próximos de 1 indicam uma boa qualidade dos dados experimentais. A seletividade diminuiu com o aumento da cadeia carbônica do alifático. Os modelos termodinâmicos usados representaram satisfatoriamente os sistemas analisados apresentando baixos desvios e boa concordância entre as linhas de amarração.

\section{REFERÊNCIAS}

ABRAMS, D. S.; PRAUSNITZ, J. M. Statistical Thermodynamics of Liquid Mixtures: A New Expression for the Excess Gibbs Energy of Partly or Completely Miscible Substances. AIChE J., v. 21, p. 116-128, 1975.

ASHOUR, I.; ABU-EISHAH, S. I. Liquid-Liquid Equilibria of Ternary and Six-Component Systems Including Cyclohexane, Benzene, Toluene, Ethylbenzene, Cumene, and Sulfolane at 303.15 K. J. Chem. Eng. Data, v. 51, p. 1717-1722, 2006a.

ASHOUR, I.; ABU-EISHAH, S. I. Liquid-Liquid Equilibria for Cyclohexane + Ethylbenzene + Sulfolane at $(303.15,313.15$, and 323.15) K. J. Chem. Eng. Data, v. 51, p. 859-863, $2006 b$.

CHEN, J.; DUAN, L. P.; MI, J. G.; FEI, W. Y.; LI, Z. C. Liquid-Liquid Equilibria of Multicomponent Systems including Hexane, Octane, Benzene, Toluene, Xylene and Sulfolane at 298.15K and Atmospheric Pressure. Fluid Phase Equil., v. 173, , 2000a.

CHEN, J.; LI, Z. C.; DUAN, L. P. Liquid-Liquid Equilibria of Ternary and Quaternary Systems including Cyclohexane, 1-Heptene, Benzene, Toluene and Sulfolane at 298.15 K. J. Chem. Eng. Data, v. 45, p. 689-692, 2000 b.

FREDENSLUND, A. A.; GMEHLING, J.; RASMUSSEN, P. Vapour-Liquid Equilibria using UNIFAC. Elsevier, Amsterdam, 1977.

LETCHER, T. M.; REDHI, G. G.; RADLOFF, S. E.; DOMANSKA, U. Liquid-Liquid Equilibria of the Ternary Mixtures with Sulfolane at 303.15 K. J. Chem. Eng. Data, v. 41, p. 634-638, 1996.

LIN, W. Liquid-Liquid Equilibria of Alkane (C10/C14) + sec-Butylbenzene + Sulfolane. $J$. Chem. Eng. Data, v. 56, p. 3122-3127, 2011.

MESQUITA, F. M. R.; FEITOSA, F. X; SOMBRA N. E.; AGUIAR, R. S. S.; SANT'ANA, H. B. Liquid-Liquid Equilibrium for Ternary Mixtures of Biodiesel (Soybean or Sunflower) + Glycerol + Ethanol at Different Temperatures. J. Chem. Eng. Data, v. 56, p. 4061-4067, 2011.

NELDER, J. A.; MEAD, R. A. A simplex method for function minimization. Comp. J., v.7, n. 4, p. 308-313, 1965.

OTHMER, D. F.; TOBIAS, P. E. Tie line correlation. Ind. Eng. Chem., v. 34, n. 6, p. 693696, 1942.

RENON, H; PRAUSNITZ, J. M. Local Compositions in Thermodynamics: Excess Functions for Liquid Mixtures. AIChE J., v. 14, p. 135-144, 1968.

SANDLER, S. I. Chemical, biochemical, and engineering thermodynamics. New York: John Wiley \& Sons Inc., p. 960, 2006.

SEVGILI, L. M. Liquid phase equilibria for mixtures of (an aliphatic hydrocarbon+ toluene $+\gamma$-butyrolactone) at $298.2 \mathrm{~K}$ and atmospheric pressure. Fluid Phase Equil., v. 287, 2010. 